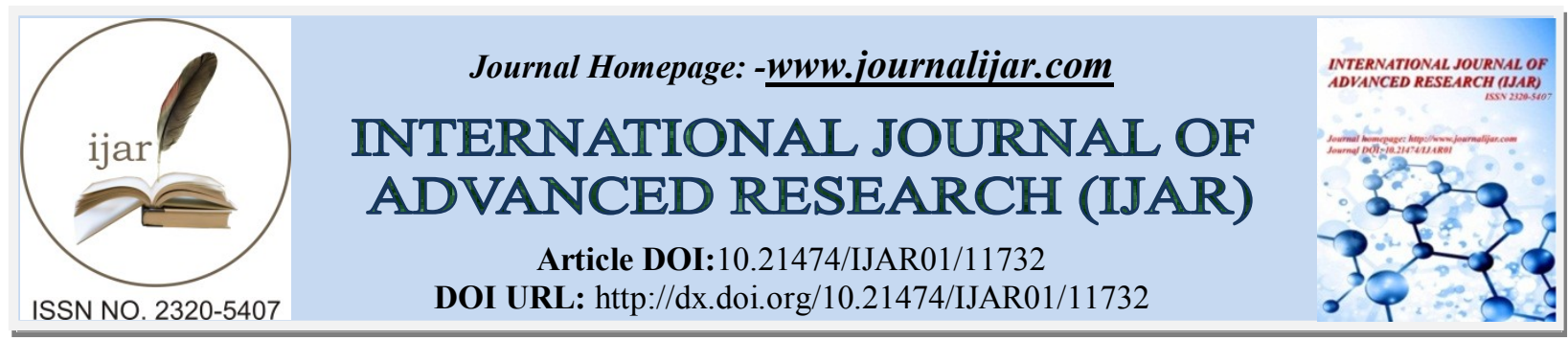

RESEARCH ARTICLE

\title{
A STUDY TO ASSESS THE EFFECT OF NURSING INTERVENTIONS REGARDING INTER COSTAL DRAINAGE CARE IN A VIEW TO PREPARE A PROTOCOL FOR NURSES.
}

\author{
Mr. Prashant Pradip Malap \\ M.Sc Nursing, Lecturer, College of Nursing, Wanless Hospital, MMC, Miraj.
}

\section{Manuscript Info}

(..........................

Manuscript History

Received: 15 July 2020

Final Accepted: 18 August 2020

Published: September 2020

Key words:-

Assess, Nursing Intervention, Inter

Costal Drainage Care, Protocol

\section{Abstract}

A descriptive study was done to assess the effect of Nursing Interventions regarding Inter Costal Drainage Care in a view to prepare a protocol for Nurses' The present study was done by using Survey Method to assess the effect of nursing interventions regarding inter costal drainage care. As per the 'Dorothy Orem Self Care Deficit', a conceptual framework was made. Samples were selected from the hospital by Non-Probability Purposive Sampling Method. The sample consisted of 60 patients. The study included demographic variable such as age, gender, duration of hospitalization, previous knowledge about disease condition. And observation check list and numerical pain scale. At the beginning of the research a survey was done and the patients with intercostal drainage care was introduced by the investigator. They were explained about the purpose of the study and were assured the about confidentiality of the information between the investigator and the respondent only. Everyday 10-15 samples were taken by the investigator before data collection the consent was taken from patients. Data was collected and analysed using Descriptive and Inferential Statistics. The level of significance was tested by using paired t-test.

Copy Right, IJAR, 2020,. All rights reserved.

\section{Introduction:-}

There are many life-threatening events that the patient undergoes in his/her own life time. Even during their stay in the hospital during the process of medical treatment they can go through many crucial situations and events. One among them is when the patient is on the Inter Costal drainage system as part of the post-operative treatment. Caring for a patient with a Chest tube is a nursing challenge. By logical system of assessment, the critical care nurse will be able to master the art of chest drainage with little difficulty. This will enhance his/her skill in assessment to ensure proper nursing care in the aspect of chest drainage.

The Thoracic Society Guidelines for the insertion of a chest drain state that in current practice chest drains are used in many different hospital settings and doctors and nurses in most specialties need to be capable of their safe insertion. Incorrect placement of a chest drain can lead to significant morbidity and even mortality. Several adverse incidents related to the insertion and management of chest drains, the need for Trust guidance was identified. These guidelines are aimed at the insertion and care of chest drains in the adult patient in a hospital environment.

A survey was conducted about major complications of intercostal chest drainage insertion. In this survey a Questionnaire was sent to 148 acute hospital trust, enquiring about current practice and any adverse incidents related 
to chest drains from 2003-2008.67\% of responding trusts had encountered major complications of intercostals drainage such as haemorrhage, infection, lung re-expansion and pulmonary oedema. Thus, the survey raised the importance of training health care staff regarding chest tube management

\section{Problem Statement}

A study to assess the effect of Nursing Intervention regarding Inter costal drainage care in a view to prepare a protocol for Nurses working in selected hospitals.

\section{Objectives:-}

1. To evaluate effect of Nursing Interventions regarding Inter costal drainage care.

2. To prepare a protocol for Nurses working in selected hospitals.

\section{Assumptions}

1. Inter costal drainage care may vary from Nursing staff to other nursing staff.

2. There can be chance of infection.

\section{Methodology:- \\ Research approach:}

Survey approach

Research design:

Cross sectional research design.

Setting of the study:

Dr. D Y Patil Hospital Pimpri Pune

\section{Research variables}

1. Dependent variables: In the present study, independent variable was Nursing intervention.

2. Independent variables: In present study, dependent variables were inter costal drainage care.

\section{Demographic variables:}

This included age, gender, duration of hospitalization, Previous knowledge about disease condition.

\section{Target Population:}

The target population is aggregate of cases about which the researcher would like to generalize.

\section{Accessible Population}

The accessible population for this study were the subjects having intercostal drainage.

\section{Sample Size}

The sample size of the present study was 60 subjects with intercostal drainage.

\section{Sampling Technique}

In this study purposive sampling technique was adopted to select the subjects. Purposive sampling is a nonprobability sampling technique where subjects were chosen to be part of the sample with a specific purpose in mind and according to the study objectives.

\section{Criteria for Samples Selection}

\section{Inclusion Criteria}

Subjects who The patients admitted with inter costal drainage .

1. Those who understood English, Hindi, Marathi.

\section{Exclusion Criteria}

1. Those who were not willing to participate 
2. Those who were not willing to participate

\section{Description of Tool}

Part I: Socio demographic variables.

Information on socio demographic variable of the subjects contains three items, which included Age, Gender, Duration of hospitalization, Previous knowledge of disease condition.

Part II: Observation check list.

Part III: Numerical Pain Scale.

\section{Reliability}

In this study, the reliability of the tool has been assessed by the bio- medical engineer of stethoscope and sphygmomanometer and reliability was assessed by using Inter rator Reliability Method. Cohen's Kappa test was used and reliability result was found 0.98 . Hence, the tool is reliable.

\section{Data collection procedure}

Official permission was obtained from the authorites from the Dr. D. Y. Patil Hospital Sant Tukaram Nagar, Pimpri Chinchwad Pune 18. The data was collected at Dr. D. Y. Patil hospital for for 3 week period at data was collected for 5 days At the starting of the session they were explained about the purpose of the study and were assured about confidentiality of the information between the investigator and the respondent. The data collection was done for 5 days each Everyday 10-15 samples were taken by investigator. Before data collection the consent was taken from patients. After permission, investigator started the procedure for data collection. Each sample took 15-20 minutes for data collection. Data is collected from 60 sample.

\section{Plan for data analysis:}

The data obtained was analyzed using descriptive and inferential statistics based on objectives.

\section{Results:-}

\section{Section I:}

Description of samples (patients) based on their personal characteristics in terms of frequency and percentage

Table 1:- Description of samples (patients) based on their personal characteristics in terms of frequency and percentagen $=60$.

\begin{tabular}{|c|c|c|}
\hline Demographic variable & Freq & $\%$ \\
\hline \multicolumn{3}{|l|}{ Age } \\
\hline 18 to 30 years & 9 & $15.0 \%$ \\
\hline 31 to 40 years & 16 & $26.7 \%$ \\
\hline 41 to 50 years & 23 & $38.3 \%$ \\
\hline 50 and above & 12 & $20.0 \%$ \\
\hline \multicolumn{3}{|l|}{ Gender } \\
\hline Male & 54 & $90.0 \%$ \\
\hline Female & 6 & $10.0 \%$ \\
\hline \multicolumn{3}{|c|}{ Duration of Hospitalization } \\
\hline 1 to 5 days & 7 & $11.7 \%$ \\
\hline 5-10 days & 39 & $65.0 \%$ \\
\hline 10 days and above & 14 & $23.3 \%$ \\
\hline \multicolumn{3}{|c|}{ Previous Knowledge of respiratory disease } \\
\hline Yes & 2 & $3.3 \%$ \\
\hline No & 58 & $96.7 \%$ \\
\hline
\end{tabular}

Table No 1 shows that $15 \%$ of the patients had age $18-30$ years, $26.7 \%$ of them had age $31-40$ years, $38.3 \%$ of them had age $41-50$ years and $20 \%$ of them had age 50 years and above. $90 \%$ of them were of male gender and 10 percent were of female gender. And it shows that $11.7 \%$ patients were admitted for 1 to 5 days and $65.0 \%$ were admitted for 
5 to 10 days and $23.3 \%$ of the patient were admitted for 10 days and above. And only $3.3 \%$ of the patient had the previous knowledge about hospitalization and remaining had no any previous knowledge about it.

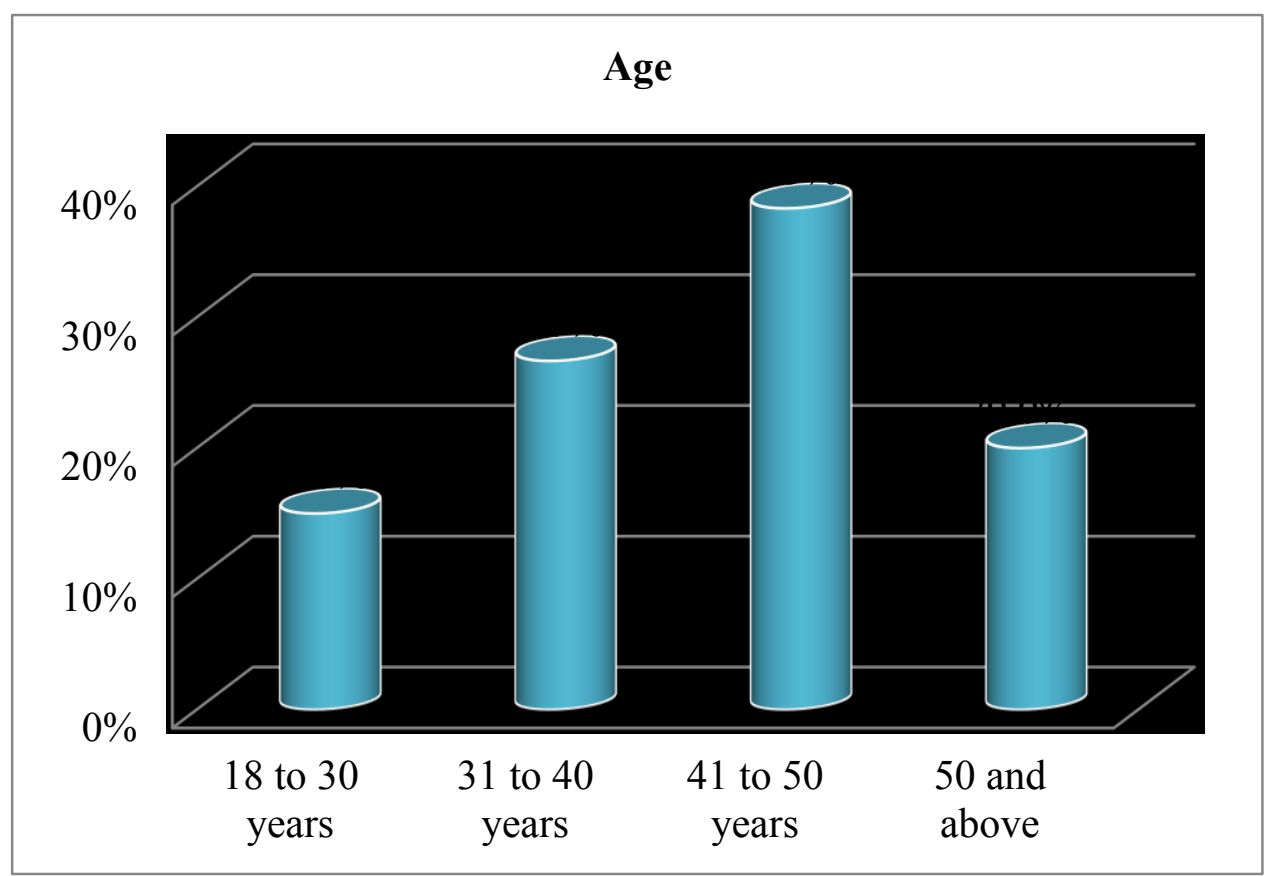

The above graph showcases that, according to age $15 \%$ of the patients had age $18-30$ years, $26.7 \%$ of them had age $31-40$ years, $38.3 \%$ of them had age $41-50$ years and $20 \%$ of them had age 50 years and above.

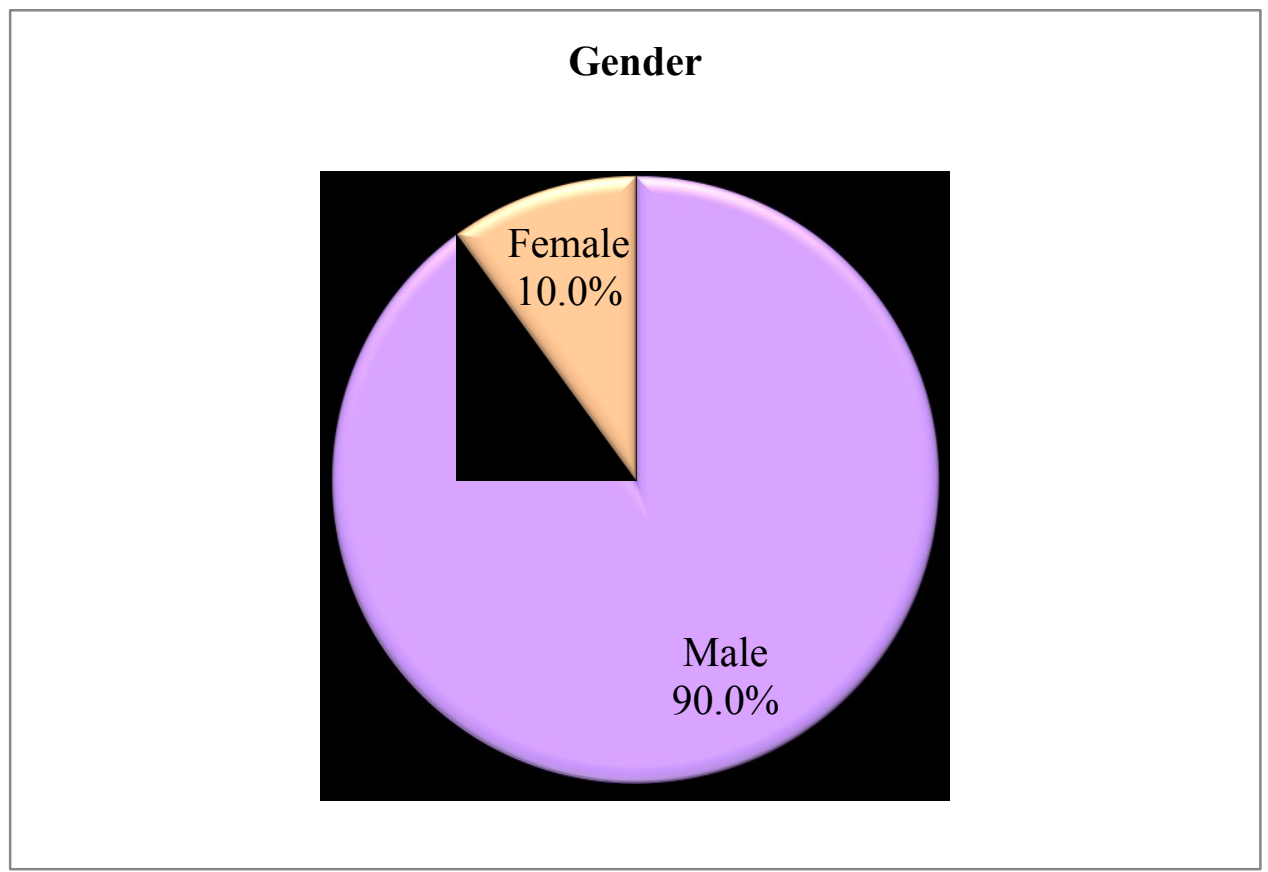

The above graph showcases that, according to gender majority of $90 \%$ of the patients were males and $10 \%$ of them were females. 


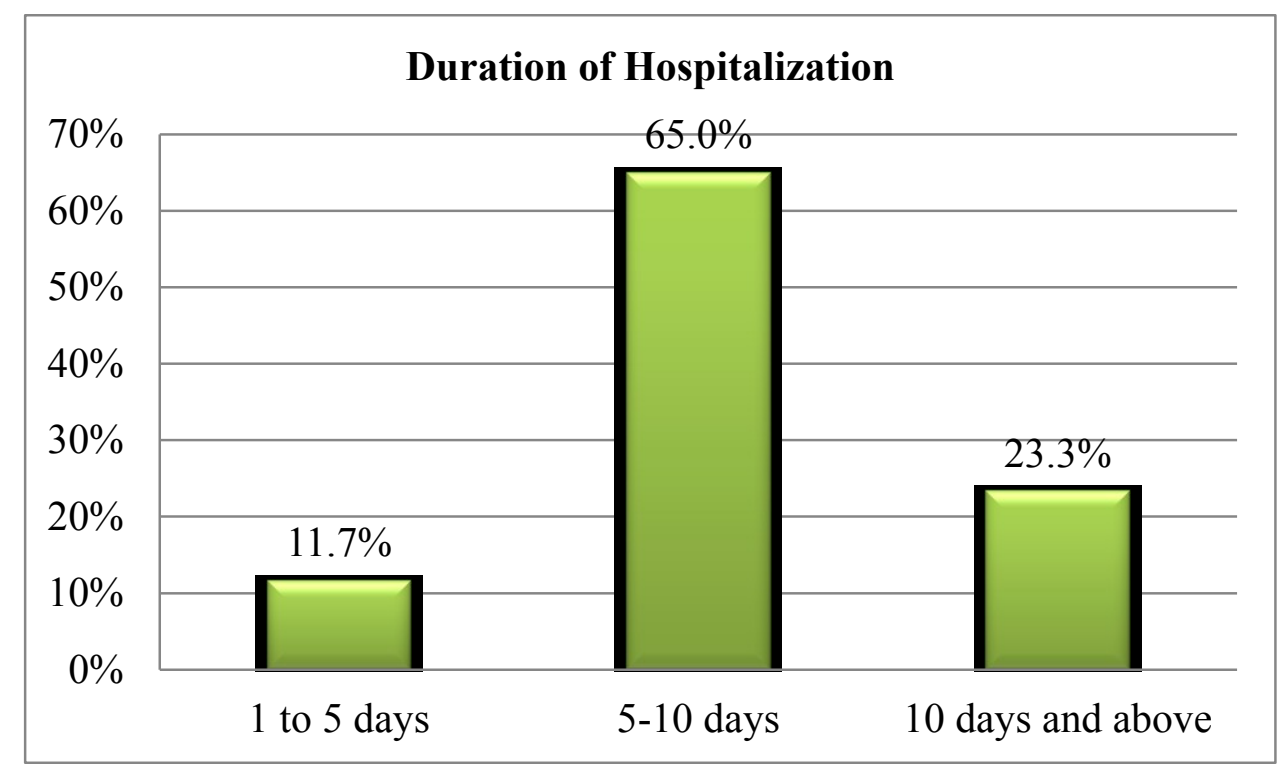

The above graph showcases that, according to duration of hospitalization mostly $65 \%$ of them had hospitalization for 5 to 10 days, $11.7 \%$ of them had hospitalization for 1 to 5 days and $23.3 \%$ of them were hospitalized for more than ten days.

\section{Previous Knowledge of Disease condition.}

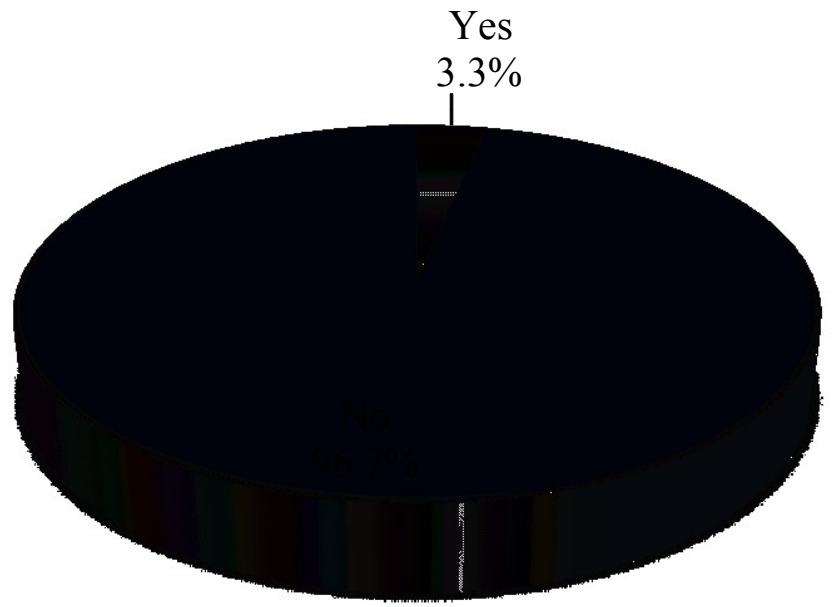

The above graph showcases that, according to previous knowledge regarding disease condition mostily had 3.3\% of them had previous knowledge of disease condition.

\section{Section II}

Analysis of data related to the effect of Nursing Intervention regarding Inter costal drainage care

$$
\mathrm{n}=60
$$

Table 2:- Effect of Nursing Intervention regarding Inter costal drainage care on respiratory rate

\begin{tabular}{|l|c|c|c|c|c|}
\hline Admin & Mean & SD & T & DF & p-value \\
\hline Day I & 26.8 & 1.2 & & & \\
\hline Day II & 26.2 & 1.4 & 4.1 & 59 & 0.000 \\
\hline Day III & 25.8 & 1.5 & 5.9 & 59 & 0.000 \\
\hline Day IV & 25.8 & 1.5 & 5.9 & 59 & 0.000 \\
\hline Day V & 25.3 & 1.6 & 7.4 & 59 & 0.000 \\
\hline
\end{tabular}


Researcher applied paired t-test for assessment of the effect of Nursing Intervention regarding Inter costal drainage care on patients. Average respiratory rate was 26.8, 26.2, 25.8, 25.8 and 25.3. T-values for this comparison were 4.1, 5.9, 5.9 and 7.4 with 59 degrees of freedom. Corresponding p-values were of the order of 0.000 which are small (less than 0.05), Nursing Intervention regarding Inter costal drainage care is proved to be significantly effective in improving the respiratory rate.

Table 3:- Effect of Nursing Intervention regarding Inter costal drainage care on respiratory rhythm

\begin{tabular}{|c|c|c|c|c|}
\hline \multirow[t]{2}{*}{ Admin } & \multicolumn{2}{|c|}{ Irregular } & \multicolumn{2}{|c|}{ Regular } \\
\hline & Freq & $\%$ & Freq & $\%$ \\
\hline Day I & 56 & $93.3 \%$ & 4 & $6.7 \%$ \\
\hline Day II & 37 & $61.7 \%$ & 23 & $38.3 \%$ \\
\hline Day III & 22 & $36.7 \%$ & 38 & $63.3 \%$ \\
\hline Day IV & 17 & $28.3 \%$ & 43 & $71.7 \%$ \\
\hline Day V & 9 & $15.0 \%$ & 51 & $85.0 \%$ \\
\hline
\end{tabular}

On day 1 , only $6.7 \%$ of the patients had regular rhythm. On day $2,38.3 \%$ of them had regular rhythm. Rhythm became regular for $63.3 \%, 71.7 \%$ and $85 \%$ of the patients on day 3 , day4 and day5. This indicates that there is remarkable improvement in the rhythm of the patients after nursing intervention regarding inter costal drainage care.

Table 4:- Effect of Nursing Intervention regarding Inter costal drainage care on respiratory depth.

\begin{tabular}{|l|r|r|r|r|r|r|}
\hline \multirow{2}{*}{ Admin } & \multicolumn{2}{|c|}{ Deep } & \multicolumn{2}{c|}{ Normal } & \multicolumn{2}{c|}{ Shallow } \\
\cline { 2 - 8 } & \multicolumn{1}{|l|}{ Freq } & \multicolumn{1}{c|}{ \% } & Freq & \% & Freq & \% \\
\hline Day I & 26 & $43.3 \%$ & 8 & $13.3 \%$ & 26 & $43.3 \%$ \\
\hline Day II & 20 & $33.3 \%$ & 20 & $33.3 \%$ & 20 & $33.3 \%$ \\
\hline Day III & 13 & $21.7 \%$ & 35 & $58.3 \%$ & 12 & $20.0 \%$ \\
\hline Day IV & 9 & $15.0 \%$ & 45 & $75.0 \%$ & 6 & $10.0 \%$ \\
\hline Day V & 6 & $10.0 \%$ & 49 & $81.7 \%$ & 5 & $8.3 \%$ \\
\hline
\end{tabular}

On day 1 , only $13.3 \%$ of the patients had normal depth of respiration. On day $2,33.3 \%$ of them had normal depth of respiration. Depth of respiration normal for $58.3 \%, 75 \%$ and $81.7 \%$ of the patients on day3, day 4 and day5. This indicates that there is remarkable improvement in the depth of respiration of the patients after nursing intervention regarding inter costal drainage care.

Table 5:- Effect of Nursing Intervention regarding Inter costal drainage care on respiratory sound.

\begin{tabular}{|c|c|c|c|c|}
\hline \multirow[t]{2}{*}{ Admin } & \multicolumn{2}{|c|}{ Abnormal } & \multicolumn{2}{|c|}{ Normal } \\
\hline & Freq & $\%$ & Freq & $\%$ \\
\hline Day I & 51 & $85.0 \%$ & 9 & $15.0 \%$ \\
\hline Day II & 38 & $63.3 \%$ & 22 & $36.7 \%$ \\
\hline Day III & 22 & $36.7 \%$ & 38 & $63.3 \%$ \\
\hline Day IV & 13 & $21.7 \%$ & 47 & $78.3 \%$ \\
\hline Day V & 8 & $13.3 \%$ & 52 & $86.7 \%$ \\
\hline
\end{tabular}

On day 1 , only $15 \%$ of the patients had normal sound of respiration. On day $2,36.7 \%$ of them had normal sound of respiration. Sound of respiration became normal for $63.3 \%, 78.3 \%$ and $86.7 \%$ of the patients on day 3 , day 4 and day5. This indicates that there is remarkable improvement in the respiration sound of the patients after nursing intervention regarding inter costal drainage care.

Table 6:- Effect of Nursing Intervention regarding Inter costal drainage care on pulse rate

\begin{tabular}{|l|c|c|c|c|c|}
\hline Admin & Mean & SD & T & df & p-value \\
\hline Day I & 75.4 & 2.0 & & & \\
\hline Day II & 74.8 & 2.1 & 3.6 & 59 & 0.000 \\
\hline Day III & 74.3 & 2.0 & 5.0 & 59 & 0.000 \\
\hline Day IV & 74.2 & 2.0 & 5.1 & 59 & 0.000 \\
\hline Day V & 74.2 & 2.0 & 5.1 & 59 & 0.000 \\
\hline
\end{tabular}


Researcher applied paired t-test for assessment of the effect of Nursing Intervention regarding inter costal drainage care on patients. Average pulse rate was 75.4, 74.8, 74.3, 74.2 and 74.2. T-values for this comparison were 2.0, 2.1, 2.0, 2.0 and 2.0 with 59 degrees of freedom. Corresponding p-values were of the order of 0.000 which are small (less than 0.05), Nursing Intervention regarding Inter costal drainage care is proved to be significantly effective in improving the pulse rate.

Table 7:- Effect of Nursing Intervention regarding Inter costal drainage care on pulse rhythm

\begin{tabular}{|c|c|c|c|c|}
\hline \multirow[t]{2}{*}{ Admin } & \multicolumn{2}{|c|}{ Irregular } & \multicolumn{2}{|c|}{ Regular } \\
\hline & Freq & $\%$ & Freq & $\%$ \\
\hline Day I & 55 & $91.7 \%$ & 5 & $8.3 \%$ \\
\hline Day II & 42 & $70.0 \%$ & 18 & $30.0 \%$ \\
\hline Day III & 26 & $43.3 \%$ & 34 & $56.7 \%$ \\
\hline Day IV & 17 & $28.3 \%$ & 43 & $71.7 \%$ \\
\hline Day V & 17 & $28.3 \%$ & 43 & $71.7 \%$ \\
\hline
\end{tabular}

On day 1 , only $8.3 \%$ of the patients had regular pulse rhythm. On day $2,30 \%$ of them had regular pulse rhythm. Pulse rhythm became regular for $56.7 \%, 71.7 \%$ and $71.7 \%$ of the patients on day3, day 4 and day5. This indicates that there is remarkable improvement in the pulse rhythm of the patients after nursing intervention regarding inter costal drainage care.

Table 8:- Effect of Nursing Intervention regarding Inter costal drainage care on pulse tension.

\begin{tabular}{|c|c|c|c|c|}
\hline \multirow[t]{2}{*}{ Admin } & \multicolumn{2}{|c|}{ Abnormal } & \multicolumn{2}{|c|}{ Normal } \\
\hline & Freq & $\%$ & Freq & $\%$ \\
\hline Day I & 52 & $86.7 \%$ & 8 & $13.3 \%$ \\
\hline Day II & 40 & $66.7 \%$ & 20 & $33.3 \%$ \\
\hline Day III & 23 & $38.3 \%$ & 37 & $61.7 \%$ \\
\hline Day IV & 10 & $16.7 \%$ & 50 & $83.3 \%$ \\
\hline Day V & 6 & $10.0 \%$ & 54 & $90.0 \%$ \\
\hline
\end{tabular}

On day 1 , only $13.3 \%$ of the patients had normal tension. On day2, $33.3 \%$ of them had normal tension. $61.7 \%$, $83.3 \%$ and $90.0 \%$ of the patients had normal tension on day 3 , day 4 and day5. This indicates that there is remarkable improvement in tension of the patients after nursing intervention regarding inter costal drainage care.

Table 9:- Effect of Nursing Intervention regarding Inter costal drainage care on systolic blood pressure.

\begin{tabular}{|l|c|c|c|c|c|}
\hline Admin & Mean & SD & T & df & p-value \\
\hline Day I & 136.5 & 6.8 & & & \\
\hline Day II & 134.0 & 7.9 & 3.8 & 59 & 0.000 \\
\hline Day III & 130.8 & 7.4 & 6.1 & 59 & 0.000 \\
\hline Day IV & 129.8 & 7.0 & 6.7 & 59 & 0.000 \\
\hline Day V & 129.8 & 7.0 & 6.7 & 59 & 0.000 \\
\hline
\end{tabular}

Researcher applied paired t-test for assessment of the effect of Nursing Intervention regarding inter costal drainage care on systolic blood pressure of patients. Average systolic blood pressure was 136.5, 134.0, 130.8, 129.8 and 129.8. T-values for this comparison were 3.8, 6.1, 6.7 and 6.7 with 59 degrees of freedom. Corresponding p-values were of the order of 0.000 which are small (less than 0.05 ), Nursing Intervention regarding inter costal drainage care is proved to be significantly effective in improving the systolic blood pressure of patients.

Table 10:- Effect of Nursing Intervention regarding Inter costal drainage care on diastolic blood pressure

\begin{tabular}{|l|c|c|c|c|c|}
\hline Admin & Mean & SD & T & df & p-value \\
\hline Day I & 93.8 & 5.2 & & & \\
\hline Day II & 92.0 & 6.3 & 3.6 & 59 & 0.000 \\
\hline Day III & 89.3 & 6.9 & 5.9 & 59 & 0.000 \\
\hline Day IV & 88.0 & 6.8 & 6.5 & 59 & 0.000 \\
\hline Day V & 88.0 & 6.8 & 6.5 & 59 & 0.000 \\
\hline
\end{tabular}


Researcher applied paired t-test for assessment of the effect of Nursing Intervention regarding inter costal drainage care on diastolic blood pressure of patients. Average diastolic blood pressure was 93.8, 92.0, 89.3, 88.0 and 88.0. Tvalues for this comparison were 3.6, 5.9, 6.5 and 6.5 with 59 degrees of freedom. Corresponding p-values were of the order of 0.000 which are small (less than 0.05 ), Nursing Intervention regarding inter costal drainage care is proved to be significantly effective in improving the diastolic blood pressure of patients.

Table 11:- Effect of Nursing Intervention regarding Inter costal drainage care on SPO2.

\begin{tabular}{|l|c|c|c|c|c|}
\hline Admin & Mean & SD & T & DF & p-value \\
\hline Day I & 93.3 & 1.9 & & & \\
\hline Day II & 93.7 & 1.9 & 2.8 & 59 & 0.003 \\
\hline Day III & 94.2 & 1.7 & 3.9 & 59 & 0.000 \\
\hline Day IV & 94.2 & 1.7 & 3.9 & 59 & 0.000 \\
\hline Day V & 94.2 & 1.7 & 3.9 & 59 & 0.000 \\
\hline
\end{tabular}

Researcher applied paired t-test for assessment of the effect of Nursing Intervention regarding inter costal drainage care on SPO2ofpatients. Average SPO2was 93.3, 93.7, 94.2, 94.2 and 94.2. T-values for this comparison were 2.8, 3.9, 3.9 and 3.9 with 59 degrees of freedom. Corresponding p-values were small (less than 0.05), Nursing intervention regarding inter costal drainage care is proved to be significantly effective in improving the SPO2of patients.

Table 12:- Effect of Nursing Intervention regarding Inter costal drainage care on air leak

\begin{tabular}{|l|c|c|c|c|c|}
\hline Admin & Mean & SD & T & DF & p-value \\
\hline Day I & 4.4 & 0.7 & & & \\
\hline Day II & 4.1 & 0.9 & 3.3 & 59 & 0.001 \\
\hline Day III & 3.8 & 0.9 & 5.2 & 59 & 0.000 \\
\hline Day IV & 3.7 & 0.9 & 5.5 & 59 & 0.000 \\
\hline Day V & 3.4 & 0.7 & 7.6 & 59 & 0.000 \\
\hline
\end{tabular}

Researcher applied paired t-test for assessment of the effect of nursing intervention regarding inter costal drainage care on air leak. Average air leak was 4.4, 4.1, 3.8, 3.7 and 3.4. T-values for this comparison were 3.3, 5.2, 5.5 and 7.6 with 59 degrees of freedom. Corresponding p-values were small (less than 0.05), Nursing intervention regarding inter costal drainage care is proved to be significantly effective in reducing the air leaks.

Table 13:- Effect of Nursing Intervention regarding inter costal drainage care on drainage color.

\begin{tabular}{|c|c|c|c|c|}
\hline \multirow[t]{2}{*}{ Admin } & \multicolumn{2}{|c|}{ Abnormal } & \multicolumn{2}{|c|}{ Normal } \\
\hline & Freq & $\%$ & Freq & $\%$ \\
\hline Day I & 58 & $96.7 \%$ & 2 & $3.3 \%$ \\
\hline Day II & 47 & $78.3 \%$ & 13 & $21.7 \%$ \\
\hline Day III & 26 & $43.3 \%$ & 34 & $56.7 \%$ \\
\hline Day IV & 15 & $25.0 \%$ & 45 & $75.0 \%$ \\
\hline Day V & 10 & $16.7 \%$ & 50 & $83.3 \%$ \\
\hline
\end{tabular}

On day 1 , only $3.3 \%$ of the patients had normal drainage colour. On day $2,21.7 \%$ of them had normal drainage colour. $56.7 \%, 75 \%$ and $83.33 \%$ of the patients had normal drainage colour on day3, day4 and day5. This indicates that there is remarkable improvement in drainage colour of the patients after nursing intervention regarding inter costal drainage care.

Table 14:- Effect of Nursing Intervention regarding Inter costal drainage care on drainage consistency.

\begin{tabular}{|c|c|c|c|c|}
\hline \multirow[t]{2}{*}{ Admin } & \multicolumn{2}{|c|}{ Abnormal } & \multicolumn{2}{|c|}{ Normal } \\
\hline & Freq & $\%$ & Freq & $\%$ \\
\hline Day I & 58 & $96.7 \%$ & 2 & $3.3 \%$ \\
\hline Day II & 46 & $76.7 \%$ & 14 & $23.3 \%$ \\
\hline Day III & 25 & $41.7 \%$ & 35 & $58.3 \%$ \\
\hline Day IV & 14 & $23.3 \%$ & 46 & $76.7 \%$ \\
\hline Day V & 8 & $13.3 \%$ & 52 & $86.7 \%$ \\
\hline
\end{tabular}


On day 1 , only $3.3 \%$ of the patients had normal drainage consistency. On day $2,23.3 \%$ of them had normal drainage consistency. $58.3 \%, 76.7 \%$ and $86.7 \%$ of them had normal drainage consistency on day3, day4 and day5. This indicates that there is remarkable improvement in drainage consistency after nursing intervention regarding inter costal drainage care.

Table 15:- Effect of Nursing Intervention regarding inter costal drainage care on drainage volume.

\begin{tabular}{|c|c|c|c|c|}
\hline \multirow[t]{2}{*}{ Admin } & \multicolumn{2}{|c|}{ Abnormal } & \multicolumn{2}{|c|}{ Normal } \\
\hline & Freq & $\%$ & Freq & $\%$ \\
\hline Day I & 55 & $91.7 \%$ & 5 & $8.3 \%$ \\
\hline Day II & 46 & $76.7 \%$ & 14 & $23.3 \%$ \\
\hline Day III & 33 & $55.0 \%$ & 27 & $45.0 \%$ \\
\hline Day IV & 20 & $33.3 \%$ & 40 & $66.7 \%$ \\
\hline Day V & 17 & $28.3 \%$ & 43 & $71.7 \%$ \\
\hline
\end{tabular}

On day 1 , only $8.3 \%$ of the patients had normal drainage volume. On day $2,23.3 \%$ of them had normal drainage volume $45 \%, 66.7 \%$ and $71.7 \%$ of them had normal drainage volume on day 3 , day4 and day5. This indicates that there is remarkable improvement in drainage volume after nursing intervention regarding inter costal drainage care.

Table 16:- Effect of Nursing Intervention regarding Inter costal drainage care on drainage oscillation.

\begin{tabular}{|l|c|r|r|}
\hline Admin & Presence & Freq & $\mathbf{\%}$ \\
\hline Day I & present & 60 & $100.0 \%$ \\
\hline Day II & present & 60 & $100.0 \%$ \\
\hline Day III & present & 60 & $100.0 \%$ \\
\hline Day IV & present & 60 & $100.0 \%$ \\
\hline Day V & present & 60 & $100.0 \%$ \\
\hline
\end{tabular}

All of the patients showed drainage oscillation on day 1 through day 5.

Table 17:- Effect of Nursing Intervention regarding Inter costal drainage care on complication.

\begin{tabular}{|c|c|c|c|c|}
\hline \multirow[t]{2}{*}{ Admin } & \multicolumn{2}{|r|}{ Present } & \multicolumn{2}{|c|}{ Absent } \\
\hline & Freq & $\%$ & Freq & $\%$ \\
\hline Day I & 60 & $100.0 \%$ & & $0.0 \%$ \\
\hline Day II & 50 & $83.3 \%$ & 10 & $16.7 \%$ \\
\hline Day III & 33 & $55.0 \%$ & 27 & $45.0 \%$ \\
\hline Day IV & 23 & $38.3 \%$ & 37 & $61.7 \%$ \\
\hline Day V & 17 & $28.3 \%$ & 43 & $71.7 \%$ \\
\hline
\end{tabular}

On day 1, all of the patients had Complication. On day $2,83.3 \%$ of them had complication55\%, 38.3\% and $28.3 \%$ of them had complication on day3, day4 and day5.

\section{Section III}

Table 19:- Effect of Nursing Intervention regarding Inter costal drainage care on pain of patients.

\begin{tabular}{|l|rr|r|r|r|r|r|}
\hline \multirow{2}{*}{ Admin } & \multicolumn{3}{|c|}{ Mild pain } & \multicolumn{3}{c|}{ Moderate pain } & \multicolumn{2}{c|}{ Severe pain } \\
\cline { 2 - 9 } & Freq & & Freq & Freq & \% \\
\hline Day I & 0 & $0.0 \%$ & 27 & $45.0 \%$ & 33 & $55.0 \%$ \\
\hline Day II & 0 & & $0.0 \%$ & 35 & $58.3 \%$ & 25 & $41.7 \%$ \\
\hline Day III & 1 & & $1.7 \%$ & 48 & $80.0 \%$ & 11 & $18.3 \%$ \\
\hline Day IV & 2 & & $3.3 \%$ & 54 & $90.0 \%$ & 4 & $6.7 \%$ \\
\hline Day V & 3 & & $5.0 \%$ & 56 & $93.3 \%$ & $1.7 \%$ \\
\hline
\end{tabular}

On day 1 , more than half (55\%) of the patients had severe pain and $45 \%$ of them had moderate pain. On day 2 , majority of $58.3 \%$ of them had moderate pain and $41.7 \%$ of them had severe pain. On day3, majority of $80 \%$ of them had moderate pain and $1.7 \%$ of them had mild pain and $18.3 \%$ of them had severe pain. On day4, majority of $90 \%$ of them had moderate pain and $6.7 \%$ of them had severe pain. On dya5, $93.3 \%$ of them had moderate pain and 
only $1.7 \%$ of them had severe pain. This shows that nursing intervention regarding inter costal drainage care was effective in improving (reducing) the pain among patients.

\section{Discussion:-}

This chapter discusses the major findings of the study and reviews them in relation to findings from the results of other studies. The aim of this study was to assess the effect of nursing interventions regarding inter costal drainage care. It shows there is significant effect on inter costal drainage care. This indicates that the there is aeffect of nursing interventions on inter costal drainage care.

\section{Nursing Practice}

Improve the practices of inter costal drainage care is an issue about which there has been a growing concern throughout the hospital etc. The primary prevention consists of two major types of activities technological and cognitive. Cognitive activities encompass health education, which is an important nursing responsibility. Nurses working in various settings like hospitals, community health, should make use of the opportunity for providing the correct information regarding practices of Inter costal drainage care. The nurses working in hospital play an important role in education about the inter costal drainage care and early detection of future complications. The nurses can conduct various Inservice education and formulate various health educations programme in various settings in the hospital.

\section{Nursing Education}

With changing health care trends nursing education must emphasize primary health care approach focusing on prevention than cure and promotion of health. The education curriculum must include imparting knowledge about the use of various teaching strategies and principles for effective planned health education. Nowadays much importance is given to awareness and promotion of health than the curative aspects. As the needs of society are continuously changing newer components must be incorporated in the nursing curriculum. Nursing education must emphasize on primary prevention and rehabilitative aspects. The basic training of nurses in India includes teaching of certain units related to care of patients with inter costal drainage care. Theoretically the focus should be on theencouragement of the nursing interventions. The nursing teachers can use the result of the research study as an informative illustration for the students. Nursing education should help in inculcating values and a sense of responsibility in the students to educate them to care for inter costal drainage care. However,specialty oriented courses are offered at the master level. Training programmes for nurses, however, need to be improved, to meet the new demands and newtrends about the inter costal drainage care practices.

\section{Nursing Administration}

In the event of ever-changing disease manifestations, knowledge explosion, technological and ever growing challenges. The administrator has a responsibility to provide nurse with substantial continuing educational opportunities. This will enable the nurses in updating their knowledge, acquiring special skills and demonstrating high quality care by deputing them for in service education programs, special courses, workshops and conferences can be arranged and attended by nursing staff in the Hospital area. Necessary administrative support should be provided for the development of such educational materials. Nursing personnel should be motivated to devote their time for development of educational material such as posters, pamphlets, and planned health teaching programme for nurses to provide a better nursing care. There is a genuine need for continuing education for nurses, particularly for those who are working in hospital. As part of administration, the nurse administrator plays a vital role in educating nurses, and student nurses. The finding of the study should be used as a basis of in-service education programs for nurses so as to make them aware of the present problems

\section{Nursing Research}

Nursing research is an essential aspect of nursing as it uplifts the profession and develops new nursing norms and a body of knowledge. Another research has been added to the Nursing literature. Many studies have done on this similar study. The research design, finding and the tool can be used as avenues for further research.

There is a need for extensive and intensive nursing research in this area. The nurse researcher should be able to conduct the research on various aspects of awareness about maintenance of intercostals care, so as to generate more scientific data. Findings of this will provide baseline data about the inter costal drainage care, and strategies that can be adopted to educate people about encourage the exclusive effect of nursing interventions regarding inter costal drainage care and it can be used for further research. 


\section{Conclusion:-}

The above study was a good learning experience for the investigator. The result of this study showed that the effect of nursing intervention was adequate, yet to meet the standard a protocol was developed and implemented for nurses who are working with patients with intercostal drainage care.

\section{Reference:-}

1. P. Fenninger John. Procedures for Primary Care Physicians. Ed $6^{\text {th }}$. St. Louis: Mosby year book 2004(11);112345.

2. Laith Farid Gulli MBBS. MS American Thoracic Society Homepage Website Addresshttp://www.thoracic.org.(3)

3. Surgery Encyclopaedia. Gale Encyclopaedia of Surgery. 2005 Dec; 67 :167-72.

4. Davies CWH, Gleeson FV, Davies RJO.BTS Guidelines. Management of Pleural Infection. British Medical Journal 2003; $58: 18-20 .(13)$

5. Ann Trop. Journal of Paediatric. Aligarh-India. 1999 March; 8 :18-21.(12)

6. Wilson, Barnett J. Assessment of Recovery with Special Reference to a Study with Post Operative Cardiac Patients. Spontaneous pneumothorax in children--a review of 95 cases. Journal of Advanced Nursing 2001:43545.

7. Richard .W .Light. Prevalence and Clinical Course of Pleural Effusions at 30 Days after Coronary Artery and Cardiac Surgery. American Journal of Critical Nursing and Management Articles 2002 Oct; 10(8) :1164.

8. Demirhan R, Ulus TravmaAcilCerrahi. Spontaneous pneumothorax: retrospective analysis of 348 cases. 2009July; 15 (15) :367-70.

9. Celik B, Sahin. Iatrogenic pneumothorax [IPnx]: Etiology, incidence and risk factor. Journal of Thoracic and Cardiovascular Surgery. 2009Aug; $57: 286-90$.

10. Kollef. Prevention of Complication among Patients with Tension Pneumothorax. Nursing management of chest drains: a systematic review. Journal of Clinical Medicine. 2006; 76(4):908-40. 